\title{
Review of series-parallel models for calculating the thermal conductivity of soils
}

\author{
Agnieszka Drzyzga ${ }^{1 *}$ \\ ${ }^{1}$ Faculty of Environmental and Power Engineering, Cracow University of Technology, al. Jana Pawła II 37, 31-864 Kraków, Poland
}

\begin{abstract}
The paper presents mixed models collected from the literature for calculating the thermal conductivity of the soil. They are created on the basis of combining the serial and parallel model. The thermal conductivity of the soil is the basic thermal parameter of the soil. Knowledge of it is necessary, among other things, for the proper design of underground infrastructure. The combination of models will help you to choose the method of calculating the thermal conductivity of the soil that gives the most accurate results and has the lowest error.
\end{abstract}

\section{Introduction}

One of the basic thermal parameters of soil is its thermal conductivity. Knowledge of it is essential for the proper design of, among others, underground infrastructure. Correct determination of this parameter is important when designing installations using renewable energy sources, operating on the basis of ground heat exchangers. There are many models for calculating the thermal conductivity of soil, available in the literature including empirical models (e.g. Kersten, 1949; Johansen, 1975; Campbell, 1985; Cote and Konrad, 2005, 2005a; Balland and Arp, 2005; Lu et al., 2007; Chen, 2008; Lu et al., 2014; Zhang et al., 2015; Tarnawski and others, 2016; Tong et al., 2016; He et al., 2017; Zhao et al., 2019; Wang et al., 2019; Tian et al., 2020; Xiao et al., 2020; Song et al., 2020; Sun et al., 2020; He et al. 2020), mathematical (e.g. De Vries, 1963; Haigh, 2012; Ofrikhter et al., 2018; Zhu, 2020), numerical (e.g. Wien et al., 2020; Rizvi et al., 2020; Zhang et al. 2020; Shrestha and Wuttke, 2020) and mixed. The compilation of the existing models was undertaken, among others, by Farouki [1], He et al. [2], Rerak [3], Zhang [4] and Różański [5]. The purpose of this article is to compile the mixed models for calculating the thermal conductivity of the soil.

\section{Review of mixed models of thermal conductivity of soil}

Mixed models are constructed on the basis of combining a serial and a parallel model. The series model assumes that a constant heat flux flows through each soil element connected in series and thus, depending on the thermal conductivity of the individual elements, each of them produces different temperature gradients. In parallel models, it is assumed that the temperature gradient in individual phases or elements is identical (each phase has the same temperature difference) but, depending on the thermal conductivity of each element, conducts a different heat flow. Series and parallel models are also referred to as upper and lower limits [6].

\subsection{The model of Wiener [7]}

Wiener derived equations indicating that the thermal conductivity of a porous medium, which consists of a soil skeleton, liquid and gas, has a lower and an upper limit. When all soil components are arranged in series, the soil reaches the lower limit, i.e. the lowest thermal conductivity, while when the soil components are arranged in parallel, the soil reaches the highest value of thermal conductivity, i.e. the upper limit. The effective thermal conductivity of mixtures arranged in parallel is given by the equation:

$$
k=k_{W}^{L}=\left[\sum \frac{n_{\alpha}}{k_{\alpha}}\right]^{-1},
$$

where $\mathrm{k}^{\mathrm{L}} \mathrm{W}$ is the thermal conductivity corresponding to the lower Wiener limit $[\mathrm{W} / \mathrm{mK}], \mathrm{n}_{\alpha}$ and $\mathrm{k}_{\alpha}$ are the porosity and thermal conductivity of the phase, respectively. For parallel mixtures, the effective thermal conductivity is given by the equation:

$$
k=k_{W}^{U}=\sum n_{\alpha} k \alpha,
$$

where $\mathrm{k}^{\mathrm{U}}$ is the thermal conductivity corresponding to the Wiener upper limit $[\mathrm{W} / \mathrm{mK}]$. Wiener boundaries are independent of the pore structure of the porous medium. This model is not applicable to soils [7].

* Corresponding author: agnieszka.drzyzga@,doktorant.pk.edu.pl 


\subsection{The model of Hashin-Shtrikman [Błąd! Nie zdefiniowano zakładki.]}

According to Dong et al. [6] the Hashin-Shtrikman model sets the effective limits of thermal conductivity for composite materials. The upper HS_U and lower HS-L limits of the model always lie within the parallel / series limits, regardless of the component volumetric fraction or thermal conductivity. The lower limit of the model is determined by the equations:

$$
k_{H S}^{L}=k_{1}+3 k_{1} \frac{\sum_{i=2}^{3}\left(f_{i} /\left(1+c_{i}^{L}\right)\right)}{f_{1}+\sum_{i=2}^{3}\left(f_{i} c_{i}^{L} /\left(1+c_{i}^{L}\right)\right)^{\prime}}
$$

where $f$ is the volumetric fraction of individual phases: air, water, soil skeleton [-], and

$$
c_{i}^{L}=3 k_{1} /\left(k_{i}-k_{1}\right),
$$

where $\mathrm{k}_{\mathrm{i}}$ is the thermal conductivity of the individual phases $[\mathrm{W} / \mathrm{mK}]$ and $\mathrm{k}_{\mathrm{a}}=0,56, \mathrm{k}_{\mathrm{w}}=0,026$. The upper limit is described by the equation:

$$
k_{H S}^{U}=k_{3}+3 k_{3} \frac{\sum_{i=1}^{2}\left(f_{i} /\left(1+c_{i}^{U}\right)\right)}{\phi_{3}+\sum_{i=1}^{2}\left(f_{i} c_{i}^{U} /\left(1+c_{i}^{U}\right)\right)^{\prime}}
$$

where:

$$
c_{i}^{U}=3 k_{3} /\left(k_{i}-k_{3}\right) .
$$

The model assumes that the composite materials are macroscopically homogeneous, isotropic, multiphase.

\subsection{The model of Tong et al. [8]}

Based on the Wiener model Tong et al. [8] proposed a model of thermal conductivity in a closed form. It takes into account the combined effect of the mineral composition of the soil skeleton, temperature, degree of liquid saturation, porosity and pressure on the effective thermal conductivity of porous media in a multiphase flow with a phase change. The effective thermal conductivity is calculated in three stages: in the first step, the thermal conductivity of the individual soil components, i.e. gas, liquid (water) and the soil skeleton, is calculated; then the effective thermal conductivity of the two-phase soil skeleton - gas mixture is determined, in the third step the effective thermal conductivity of the three-phase mixture of solid, liquid and gas is determined. The model strictly adheres to Wiener and Hashin-Shtrikman constraints when the porosity $\mathrm{n}$ is in the range $[0.04 ; 0.97]$ and the saturation degree with $\mathrm{Sr}$ is in the range $[0,1]$. The effective thermal conductivity of the whole mixture is expressed as:

$$
k=\left(1-\eta_{2}\right) k^{L}+\eta_{2} k^{U},
$$

where $\eta_{2}$ is a function of the pore structure, saturation and temperature and according to Wiener constraints for anisotropic mixtures it should belong to the range $[0,1]$, $\mathrm{k}^{\mathrm{L}}$ and $\mathrm{k}^{\mathrm{U}}$ are the upper and lower limits of the threephase mixture. According to the Wiener limits $\mathrm{k}^{\mathrm{L}}$ for series connections between solid, liquid and gaseous phases is calculated from the formula:

$$
\begin{gathered}
k^{L}=n_{1} k_{s}+n_{2}\left[\frac{(1-n)\left(1-\eta_{1}\right)}{n_{2}} \frac{1}{k_{s}}+\frac{n S_{r}}{n_{2}} \frac{1}{k_{w}}+\right. \\
\left.\frac{n\left(1-S_{r}\right)}{n_{2}} \frac{1}{k_{g}}\right]^{-1}=\eta_{1}(1-n) k_{s}+\left[1-\eta_{1}(1-n)\right]^{2} \\
{\left[\frac{(1-n)\left(1-\eta_{1}\right)}{k_{s}}+\frac{n S_{r}}{k_{w}}+\frac{n\left(1-S_{r}\right)}{k_{g}}\right]^{-1}}
\end{gathered}
$$

where $\mathrm{kg}_{\mathrm{g}}$ is the thermal conductivity of the gas $[\mathrm{W} / \mathrm{mK}]$, $\mathrm{k}_{\mathrm{w}}$ is the thermal conductivity of water $[\mathrm{W} / \mathrm{mK}], \mathrm{k}_{\mathrm{s}}$ is the thermal conductivity of the solid phase $[\mathrm{W} / \mathrm{mK}], \eta_{1}$ is the coefficient depending on the pore structure of the solid-gas mixture and should be in the range $[0,1]$ according to Wiener limits. For parallel connections between solid, liquid and gaseous phases $\mathrm{k}^{\mathrm{U}}$ is calculated from the formula:

$$
\begin{aligned}
k^{U}= & n_{1} k_{s}+n_{2}\left[\frac{(1-n)\left(1-\eta_{1}\right)}{n_{2}} k_{s}+\frac{n S_{r}}{n_{2}} k_{w}+\right. \\
\left.\frac{n\left(1-S_{r}\right)}{n_{2}} k_{g}\right]= & \eta_{1}(1-n) k_{s}+\left[(1-n)\left(1-\eta_{1}\right) k_{s}+\right. \\
& \left.n S_{r} k_{w}+n\left(1-S_{r}\right) k_{g}\right] .
\end{aligned}
$$

The fixed phase in the above equations is divided into two parts, where $n_{1}=\eta_{1}(1-n)$ is constant due to the parallel connections and $n_{2}=1-\eta_{1}(1-n)$ occurs in a parallel / series connection. In the case of isotropic materials, the model also takes into account the HashinShtrikman rules, that is:

$$
k_{H-S}^{L} \leq k \leq k_{H-S}^{U},
$$

where the limits of the coefficient $\eta_{2}$ are:

$$
\begin{aligned}
& \eta_{2} \geq \eta_{2}^{L}=\frac{k^{L}-\eta_{1}(1-n) k_{s}-B\left[1-\eta_{1}(1-n)\right]^{2}}{A-B\left[1-\eta_{1}(1-n)\right]^{2}}, \\
& \eta_{2} \leq \eta_{2}^{U}=\frac{k^{U}-\eta_{1}(1-n) k_{S}-B\left[1-\eta_{1}(1-n)\right]^{2}}{A-B\left[1-\eta_{1}(1-n)\right]^{2}},
\end{aligned}
$$

the coefficients A and B are determined as follows:

$$
\begin{gathered}
A=(1-n)\left(1-\eta_{1}\right) k_{s}+n S_{r} k_{w}+n\left(1-S_{r}\right) k_{g}, \\
B=\left[\frac{(1-n)\left(1-\eta_{1}\right)}{k_{s}}+\frac{n S_{r}}{k_{w}}+\frac{n\left(1-S_{r}\right)}{k_{g}}\right]^{-1} .
\end{gathered}
$$

The model was developed on the basis of experimental data of bentonite, but it can also be used to determine the thermal conductivity of soils, clays and rocks.

\subsection{The model of Tarnawski and Leong [9]}

Tarnawski and Leong [9] developed a series-parallel model to assess the thermal conductivity of unsaturated soils. It assumes a one-dimensional heat flow through an elementary cubic cell of an unsaturated soil. There are three paths of heat flow, i.e. "constant contact path $\Theta_{\mathrm{sb}}$, series-parallel path of solids $\Theta_{\mathrm{s}}$ in a series configuration with a parallel path of negligible groundwater content $\mathrm{n}_{\mathrm{w}}$ and negligible soil air content $\mathrm{n}_{\mathrm{a}}$, and the path of water $\Theta_{\mathrm{w}}$ and air $\Theta_{\mathrm{a}}$ in the system serial." The thermal conductivity is determined by the equation: 


$$
\begin{gathered}
k_{S-P-S}=k_{S} \Theta_{S b}+\frac{\left(1-n-\Theta_{s b}+n_{w m}\right)^{2}}{\frac{1-n-\Theta_{S b}}{k_{S}}+\frac{n_{w m}}{k_{w} \frac{n_{w}}{n_{w m}}+k_{a}\left(1-\frac{n_{w}}{n_{w m}}\right)}}+ \\
\frac{\left(n-n_{w m}\right)^{2}}{\frac{n S_{r}-n_{w m} \frac{n_{w}}{w_{w m}}}{k_{k}}+\frac{n\left(1-S_{r}\right)-n_{w m}\left(1-\frac{n_{w}}{n_{w m}}\right)}{k_{a}}}
\end{gathered}
$$

where $\mathrm{n}_{\mathrm{wm}}$ are fluid-filled fine pores. Another variant of the model takes into account the path of water and air in a parallel configuration:

$$
\begin{gathered}
k_{S-P-P}=k_{s} \Theta_{s b}+\frac{\left(1-n-\Theta_{s b}+n_{w m}\right)^{2}}{\frac{1-n-\Theta_{s b}}{k_{S}}+\frac{n_{w m}}{k_{w} \frac{n_{w}}{n_{w m}}+k_{a}\left(1-\frac{n_{w}}{n_{w m}}\right)}}+ \\
k_{w}\left(n S_{r}-n_{w m} \frac{n_{w}}{n_{w m}}\right)+k_{a}\left[n\left(1-S_{r}\right)-n_{w m}(1-\right. \\
\left.\left.\frac{n_{w}}{n_{w m}}\right)\right] .
\end{gathered}
$$

The model was calibrated with Canadian fine, medium and coarse sands.

\subsection{The model of Tokoro et al. [10]}

Tokoro et al. [10] proposed a series-parallel model for calculating the thermal conductivity of the soil (Fig. $1,2)$ :

$$
\begin{gathered}
k=\frac{1}{R_{1}}+\frac{1}{R_{2}+R_{3}}+\frac{1}{R_{4}+R_{5}}+\frac{1}{R_{6}}=k_{s} d_{1}+ \\
\frac{1}{\frac{D_{1}}{k_{s} d_{2}}+\frac{D_{2}}{k_{w} d_{2 w}+k_{a} d_{2 a}}}+\frac{\frac{1}{D_{3}}+\frac{D_{4}}{k_{S} d_{3}}+\frac{D_{w} d_{3}+k_{a} d_{3 a}}{k_{a}}}{k_{4 a}}+k_{w} d_{4 w}+
\end{gathered}
$$

where $\mathrm{R}$ is the thermal resistance. They also presented an empirical equation based on electrical resistance:

$$
k=\alpha+\beta r^{\gamma},
$$

where $\alpha$ is treated as a soil dependent variable, $\beta, \Upsilon$ are constants and $\mathrm{r}$ is electrical resistance. This method is only applicable to land used for research.

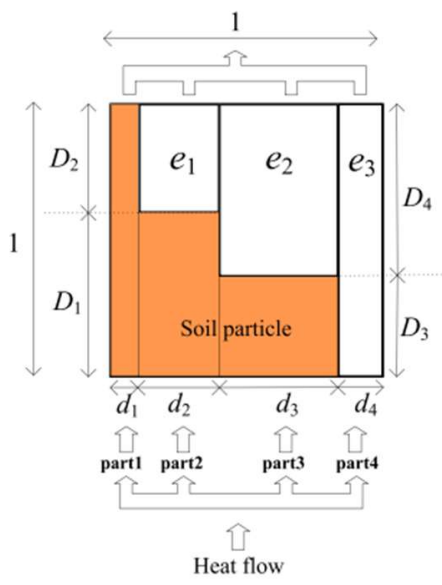

Fig. 1. Model of thermal conductivity [10]

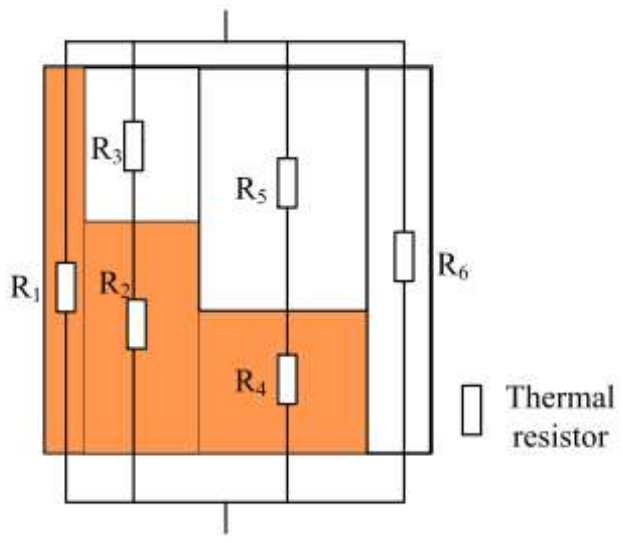

Fig. 2. Thermal resistance [10]

\subsection{The model of Lu et al. [11]}

Lu et al. (Lu et al., 2018, 2020) have proposed upper and lower limits for the thermal conductivity of soils based on the Wiener limits. They derived a simplified parallel mixed model:

$$
k=\left(f_{s} k_{s}+f_{w} k_{w}+f_{a} k_{a}\right)^{\omega} \cdot\left[\frac{1}{\left(\frac{f_{s}}{k_{s}}+\frac{f_{w}}{k_{w}}+\frac{f_{a}}{k_{a}}\right)}\right]^{1-\omega}+\zeta S_{r},
$$

where $\omega$ and $\subseteq$ are empirical fit parameters [11].

\subsection{The model of Jia et al. [12]}

Jia et al. [12] presented a three-dimensional analytical model of a packed sphere. Around the spheres lying next to each other and forming the skeleton, voids are filled with liquid and gas. The hemisphere radius reflects the actual grain diameter, and the minimum and maximum porosity of the model is 0.476 and 0.215 . The model uses soil parameters such as porosity, grain size (grain diameters), volumetric water content and thermal conductivity of individual soil components:

$$
k=\frac{\delta}{A R_{\text {total }}}=\frac{L}{2 r_{0}^{2} R_{\text {total }}}
$$

where $\mathrm{A}$ is the cross-sectional area $\left[\mathrm{mm}^{2}\right], \delta$ is the thickness [m], L is half the height of the cubic model $[\mathrm{mm}], \mathrm{r}_{0}$ is the radius of the solid particle $[\mathrm{mm}]$, and $\mathrm{R}_{\text {total }}$ is the total thermal resistance $[\mathrm{K} / \mathrm{W}]$ and $\frac{1}{R_{\text {total }}}=$ $\frac{1}{R_{1}+R_{5}}+\frac{1}{R_{2}}+\frac{1}{R_{3}}+\frac{1}{R_{4}}$, where $\mathrm{R}_{\mathrm{i}}$ is the thermal resistance of the $\mathrm{i}$-th segment $[\mathrm{K} / \mathrm{W}], \mathrm{R}_{5}$ is the contact resistance $[\mathrm{K} / \mathrm{W}]$.

\subsection{The model of Liu et al. [11]}

Liu et al. [11] proposed a modified series-parallel model applicable to clayey clays. It is based on soil parameters such as water content, dry density, degree of saturation $\mathrm{S}_{\mathrm{r}}$ and porosity $\mathrm{n}$. For silty clays, apart from the critical water content, the model is as follows: 


$$
\begin{array}{r}
k=\left(f_{s} k_{s}+f_{w} k_{w}+f_{a} k_{a}\right)^{0.58} \cdot\left[\frac{1}{\left(\frac{f_{s}}{k_{s}}+\frac{f_{w}}{k_{w}}+\frac{f a}{k_{a}}\right)}\right]^{0.42}+ \\
0.75 S_{r}+A w+B \rho_{d}+C S_{r}+D n+F,(21)
\end{array}
$$

where $\rho_{\mathrm{d}}$ is the dry density $\left[\mathrm{kg} / \mathrm{m}^{3}\right], \mathrm{A}, \mathrm{B}, \mathrm{C}, \mathrm{D}, \mathrm{F}$ are empirical coefficients. The equation for calculating the thermal conductivity takes the form

$$
\begin{gathered}
k=\left(f_{s} k_{s}+f_{w} k_{w}+f_{a} k_{a}\right)^{0.58} \cdot\left[\frac{1}{\left(\frac{f_{s}}{k_{s}}+\frac{f_{w}}{k_{w}}+\frac{f_{a}}{k_{a}}\right)}\right]^{0.42}+ \\
0.75 S_{r}-14.56 w-7.57 \rho_{d}+9.92 S_{r}+7.67
\end{gathered}
$$

when the water content in the silty clays exceeds the critical water content.

\subsection{The model of Bi et al. [13]}

Bi et al. [13] presented a generalized model for calculating the thermal conductivity of frozen soils, taking into account soil components and the frost wave that occurs during soil freezing. The soil freezing process has been divided into three stages for which generalized mixed models have been developed, and these are a function of still water content, frost heave, porosity and initial water content. The model assumes that there are both series and parallel connections between water and ice. In stage 1 there is no frost wave and the ground consists of a solid part, air, water and ice. Stage 2 is a critical state where the pores of the soil are filled only with unfrozen water and ice. There is no frost wave here. In stage 3 there is a frost wave and the ground consists of a solid part, water and ice. The generalized model takes the form:

$$
k=\left\{\begin{array}{lr}
k_{f-\text { eta }} & \theta_{u}>\theta_{u-C r i}=f_{w 0}-\frac{f_{a 0}}{(h-1)} \\
k_{f-\text { etap } 2} & \theta_{u}=\theta_{u-C r i}=f_{w 0}-\frac{f_{a 0}}{(h-1)}, \\
k_{f-\text { etap } 3} & \theta_{u}<\theta_{u-C r i}=f_{w 0}-\frac{f_{a 0}}{(h-1)}
\end{array}\right.
$$

where $k_{f-\text { etap } i}=\kappa_{\xi} k_{f \text {-etap } i}^{p}+\left(1-\xi_{j}\right) k_{f-\text { etap } i}^{s}$, where $\mathrm{k}_{\mathrm{f} \text {-etap } \mathrm{i}}$ is the thermal conductivity of frozen soils in individual stages [W/mK], $k_{f-e t a p i}^{p}$ and $k_{f-\text { eta } i}^{s}$ is the thermal conductivity of frozen soils in individual stages, assuming that ice and non-frozen water are arranged parallel and in series, respectively $[\mathrm{W} / \mathrm{mK}], \xi$ is a weighing parameter and is in the range $<1,1>$ and

$$
\begin{aligned}
k_{f-e t a}^{p} & =\eta f_{s 1} k_{s}+\eta f_{w 1} k_{w}+\eta f_{i 1} k_{i}+\eta f_{a 1} k_{a}+ \\
& (1-\eta)\left[\frac{f_{s 1}}{k_{s}}+\frac{\left(f_{w 1}+f_{i 1}\right)^{2}}{f_{w 1} k_{w}+f_{i 1} k_{i}}+\frac{f_{a 1}}{k_{a}}\right]^{-1}
\end{aligned}
$$

where $\mathrm{k}^{\mathrm{p}_{\text {f-etap } 1}}$ is the thermal conductivity of frozen land in stage 1 for the condition that ice and water are parallel [W/mK], $\mathrm{k}_{\mathrm{i}}$ is the thermal conductivity of ice [W/mK], $\mathrm{f}_{\mathrm{s} 1}, \mathrm{f}_{\mathrm{w} 1}, \mathrm{f}_{\mathrm{i} 1}, \mathrm{f}_{\mathrm{a} 1}$ are the volume fractions of solid, water, ice, and air, respectively, in step 1 and are:

$$
\begin{gathered}
f_{s 1}=f_{s 0}, f_{w 1}=\theta_{u}, f_{i 1}=h\left(f_{w 0}-\theta_{u}\right), \quad f_{a 1}= \\
f_{a 0}-(h-1)\left(f_{w 0}-\theta_{u}\right),
\end{gathered}
$$

where $\theta_{\mathrm{u}}$ is the volumetric content of the non-frozen water and $h$ is the expansion parameter of the water-ice phase transition and $\mathrm{h}=\rho_{\mathrm{w}} / \rho_{\mathrm{i}}$,

$$
\begin{gathered}
k_{f-\text { etap } 1}^{s}=\eta f_{s 1} k_{s}+\eta\left(f_{w 1}+f_{i 1}\right)^{2}\left[\frac{f_{w 1}}{k_{w}}+\frac{f_{i 1}}{k_{i}}\right]^{-1}+ \\
\eta f_{a 1} k_{a}+(1-\eta)\left[\frac{f_{s 1}}{k_{s}}+\frac{f_{w 1}}{k_{w}}+\frac{f_{i 1}}{k_{1}}+\frac{f_{a 1}}{k_{a}}\right]^{-1}, \\
k_{f-\text { etap } 2}^{p}=\eta f_{s 2} k_{s}+\eta f_{w 2} k_{w}+\eta f_{i 2} k_{i}+(1- \\
\eta)\left[\frac{f_{s 2}}{k_{s}}+\frac{\left(f_{w 2}+f_{i 2}\right)^{2}}{f_{w 2} k_{w}+f_{i 2} k_{i}}\right]^{-1}, \\
k_{f-\text { etap } 2}^{s}=\eta f_{s 2} k_{s}+\eta\left(f_{w 2}+f_{i 2}\right)^{2}\left[\frac{f_{w 2}}{k_{w}}+\frac{f_{i 2}}{k_{i}}\right]^{-1}+ \\
(1-\eta)\left[\frac{f_{s 2}}{k_{s}}+\frac{f_{w 2}}{k_{w}}+\frac{f_{i 2}}{k_{i}}\right]^{-1},
\end{gathered}
$$

where:

$$
\begin{gathered}
f_{s 2}=f_{s 0}, f_{w 2}=\theta_{u-C r i}, \quad f_{i 2}=h\left(f_{w 0}-\theta_{u-C r i}\right), \\
\text { gdzie } \theta_{u-C r i}=f_{w 0}-\frac{f_{a 0}}{(h-1)},
\end{gathered}
$$

where $\theta_{u-C r i}$ is the critical volume of unfrozen water in step 2,

$$
\begin{gathered}
k_{f-e t a p 3}^{p}=\frac{\eta f_{s 3} k_{s}}{1+\varepsilon}+\frac{\eta f_{w 3} k_{w}}{1+\varepsilon}+\frac{\eta f_{i 3} k_{i}}{1+\varepsilon}+(1- \\
\eta)\left[\frac{f_{s 3}}{(1+\varepsilon) k_{s}}+\frac{\left(f_{w 3}+f_{i 3}\right)^{2}}{(1+\varepsilon) f_{w 3} k_{w}+f_{i 3} k_{i}}\right]^{-1}, \\
k_{f-\operatorname{etap} 3}^{s}=\frac{\eta f_{s 3} k_{s}}{1+\varepsilon}+\frac{\eta\left(f_{w 3}+f_{i 3}\right)^{2}}{1+\varepsilon}\left[\frac{f_{w 3}}{k_{w}}+\frac{f_{i 3}}{k_{i}}\right]^{-1}+ \\
(1-\eta)\left[\frac{f_{S 3}}{(1+\varepsilon) k_{s}}+\frac{f_{w 3}}{(1+\varepsilon) k_{w}}+\frac{f_{i 3}}{(1+\varepsilon) k_{i}}\right]^{-1}
\end{gathered}
$$

where:

$$
f_{s 3}=f_{s 0}, f_{w 3}=(1+\varepsilon) \theta_{u}, \quad f_{i 3}=h\left[f_{w 0}-(1+\right.
$$

where $\varepsilon$ is a frost wave and $\mathrm{f}_{\mathrm{s} 3}+\mathrm{f}_{\mathrm{w} 3}+\mathrm{f}_{\mathrm{i} 3}=1+\varepsilon$.

\section{Conclusions}

The work presents 9 mixed models for calculating the thermal conductivity of soils. Despite the availability of many models described in the literature, there is no universal model that would be universally applicable. The above list, however, will make it easier to find a model that can be adapted to the specific analyzed case.

1. O.T. Farouki. Thermal properties of soils. Series Rock Soil Mech, 11:1-136 (1986).

2. H. He, D. He, J. Jin, K. M. Smits, M. Dyck, Q. Wu, B.Ch. Si, J. Lv. Room for Improvement: A Review and Evaluation of 24 Soil Thermal Conductivity Parameterization Schemes Commonly Used in Land-Surface, Hydrological, and Soil-Vegetation-Atmosphere Transfer Models. Earth-Sci Rev, 211:103419 (2020). doi:10.1016/J.EARSCIREV.2020.103419

3. M. Rerak. Selected Soil Thermal Conductivity Models. E3S Web of Conferences, 13:4-7 (2017). doi:10.1051/e3sconf/20171302003 
4. N. Zhang, Z. Wang. Review of Soil Thermal Conductivity and Predictive Models. Int J Therm Sci 117:172-83 (2017). doi: 10.1016/j.ijthermalsci.2017.03.013

5. A. Różański, N. Kaczmarek. Empirical and Theoretical Models for Prediction of Soil Thermal Conductivity: A Review and Critical Assessment. Studia Geotechnica et Mechanica, 42 (4):330-40 (2020). doi:10.2478/sgem-2019-0053

6. Y. Dong, J.S. McCartney, N. Lu. Critical review of thermal conductivity models for unsaturated soils. Geotech Geol Eng, 33 (2) (2015). doi:10.1007/s10706-015-9843-2

7. N. Zhang, Z. Wang. Review of soil thermal conductivity and predictive models. International. Journal of Thermal Sciences, 117: 172-183 (2017). doi.org/10.1016/j.ijthermalsci.2017.03.013

8. F. Tong, L. Jing, R.W. Zimmerman. An effective thermal conductivity model of geological porous media for coupled thermo-hydro-mechanical systems with multiphase flow. International Journal of Rock Mechanics \& Mining Sciences, 46: 1358-1369 (2009). doi.org/10.1016/j.ijrmms.2009.04.010

9. V.R. Tarnawski, W.H. Leong. A series -parallel model for estimating the thermal conductivity of unsaturated soils. Int J Thermophys, 33: 11911218 (2012). doi:10.1007/s10765-012-1282-1

10. T. Tokoro, T. Ishikawa, S. Shirai, T. Nakamura. Estimation methods for thermal conductivity of sandy soil with electrical characteristics. Soil and Foundations, 56 (5): 927-36 (2016). doi.org/10.1016/j.sandf.2016.08.016

11. X. Liu, G. Cai, S.S.C. Congress, L. Liu, S. Liu. Investigation of thermal conductivity and prediction model of mucky silty clay. J. Mater. Civ. Eng., 32 (8): 04020221 (2020). doi.org/10.1061/(ASCE)MT.1943-5533.0003294

12. G.S. Jia, Z.D. Ma, Y. Cao, X.Z. Meng, L.Y. Zhang, J.C. Chai, L.W. Jin. A new packed-sphere model for geological materials thermal conductivity prediction at moderate porosity range for geothermal utilization. Int J Energy Res, 1-15 (2019). doi: 10.1002/er.5067

13. J. Bi, M. Zhang, Y. Lai, W. Pei, J. Lu, Z. You, D. $\mathrm{Li}$. A generalized model for calculating the thermal conductivity of freezing soils based on soil components and frost heave. International Journal of Heat and Mass Transfer, 150:119166 (2020). doi.org/10.1016/j.ijheatmasstransfer.2019.119166 